\title{
Embarazo en adolescentes en el Hospital Universitario Metropolitano
}

\author{
Guillermo Acosta Osio*
}

\begin{abstract}
RESUMEN: Para conocer la incidencia, así como para determinar el alto riesgo obstétrico y lograr la detección precoz de las probables complicaciones con el embarazo en las adolescentes menores de 17 años, decidimos realizar un estudio descriptivo, prospectivo, incluyendo a todas las adolescentes de 17 años o menos, embarazadas, que acudieron al Hospital Universitario Metropolitano, para su control prenatal y resolución obstétrica, desde enero de 1987, hasta diciembre de 1990.
\end{abstract}

Encontramos una incidencia en nuestro medio de $13.8 \%$ de embarazo en adolescentes, de los cuales $5.8 \%$ correspondía a jóvenes de 17 años o menos, también se encontró que $\mathbf{2 5 \%}$ de las pacientes presentaron complicaciones, siendo la más frecuente el parto prematuro.

Entre las conclusiones recalcamos la importancia de que, en todas las instituciones hospitalarias debe existir una consulta especializada para atender a las adolescentes desde el punto de vista obstétrica y ginecológico, y, que esta consulta debe estar conformada por el equipo de salud, a saber: médico, psicólogo, enfermera, nutricionista, trabajadora social, etc.

PALABRAS CLAVES: Embarazo en adolescentes, complicaciones del embarazo, resultados perinatales.

SUMMARY: To be able to know the incidence, and gain some knowledge as well as to determine the high obstetric risks, and be able to detect early complications with teenage pregnancies. We decided to do a descriptive and prospective study including all teenagers from seventeen years of age and younger.

Our study, includes every teenager who was 17 and less, that came to the Metropolitana University Hospital for pre-natal care and an obstetric resolution, since january of 1987 , all thru december of 1990 .

We found an incidence in our society of $13.8 \%$ of teenage pregnancy, of which $5.8 \%$ corresponded to teenagers seventeen and younger. We also found that $25 \%$ of the patients presented complications, most often being a premature delivery.

Among our conclusions, we would like to emphasise the importance of having a specialized consulting office for adolescents at every Hospital. From the Ginecological and Obstetrics point of view, this should be conformed by a health group of doctors, pshichologists, nurses, nutricionits, social workers, etc.

KEY WORDS: Teenagers pregnancies, pregnancy complications, perinatal results.

La adolescencia, etapa confusa de la vida, por ser una época de transición entre la niñez y la adultez, se inicia con los cambios puberales y termina al adquirirse el estatus de adulto, este proceso va a depender de las condiciones socioeconómicas y culturales de cada familia. En esta época existe desarrollo de tres aspectos como son el físico, adquiriendo el hombre o la mujer su cuerpo de adulto, el socio-sexual que le va a permitir delimitar su roll en la sociedad y el psicológico que facilitará la adquisición de un pensamiento formal analítico y lograr su propia identidad (1).

Estos cambios en nuestro mundo actual, debido a la inestabilidad de las sociedades modernas, a los adelantos técnico-científicos, a los medios de comunicación, y otros,

* Profesor y Director Dpto. Ginecología y Obstetricia. Hospital Universitario Metropolitano. que son utilizados de manera indiscriminada y mercantilista aprovechándose de la incapacidad crítica de las adolescentes, favorecen el que muchos de estos no logren ajustarse adecuadamente a su roll y se confundan fácilmente; ya que incluso los cambios a nivel corporal le generan profunda angustia, que si no es bien canalizada por sus padres $\mathrm{u}$ otros adultos, puede resolverse de manera conflictiva (2), lo cual los lleva a actuar inadecuadamente, sobre todo desde el punto de vista sexual, ya que en todas partes y por todos lados, inculcan la idea que en la vida el sexo debe ser lo primero y es presentado como objeto de consumo y como punto culminante de la vida, sin que este grupo de jóvenes (y aún muchos adultos) logren tener en cuenta que existen otras prioridades en la vida; y es así, como encontramos que el $90 \%$ de los adolescentes hombres han tenido su primera relación sexual entre los 15 y 20 años, y entre las mujeres de esta edad, ya tenían experiencia sexual el $52 \%$ (3). 
Duarte (1) encontró que el embarazo entre los 11 y 17 años equivalía a un $12.2 \%$ y que esta cifra era de $1.5 \%$ hace 15 años. En el estudio de Barrios Amaya (5) en 1985, éste encontró en el Hospital de Mațernidad Rafael Calvo de Cartagena, Colombia, el inicio de vida marital de las pacientes, a los 14 años; con un promedio de inicio de vida obstétrica entre esas pacientes de 16 años; además encontró la alarmante cifra de $27.2 \%$ de complicaciones, o sea, que entre más tempranamente se inician las relaciones sexuales, mayor el riesgo de embarazo en las adolescentes.

En vista de lo anterior y de la importancia de la medicina preventiva, de la detección precoz de los padecimientos y del alto riesgo obstétrico que conlleva un embarazo en menores de 17 años, decidimos realizar este estudio, pues la adolescencia es una etapa de la vida, en la cual el proceso de cambios que se originan en la joven puede desarrollar una serie de conflictos al saberse embarazada, que llevarían desde el aborto, hasta un matrimonio forzado, entrega del hijo en adopción, o llegar hasta maltrato del recién nacido.

\section{Material y métodos}

Se realizó un estudio descriptivo, prospectivo, de las pacientes que acuden a la consulta de alto riesgo obstétrico, para embarazadas de 17 años o menos, que funciona en el área de materno-infantil, del Departamento de Ginecología y Obstetricia del Hospital Universitario Metropolitano y que es atendida por un equipo de salud conformado por enfermería, psicología, nutrición, trabajo social y médicos ginecoobstetras y pediatras, incluyendo sólo las jóvenes desde enero de 1987 hasta. diciembre de 1990, que tuvieron la resolución del embarazo en el Hospital Universitario Metropolitano, teniendo en cuenta las siguientes variables: edad, estado civil, escolaridad, lugar de residencia, menarquia, inicio de vida sexual, control prenatal, actitud al saber que estaba embarazada y los resultados obstétricos y perinatales, para luego realizar los análisis estadísticos requeridos.

\section{Resultados}

En estos cuatro años, ingresaron para atención obstétrica un total de 3.286 pacientes, de las cuales $392(12 \%)$ presentaban cuadro de aborto y entre las mismas habían 9 (2.3\%) adolescentes de 17 años o menos; las restantes 2.894 pacientes $(88 \%)$ presentaban embarazos mayores de 20 semanas y de estas, 102 pacientes (3.5\%) eran jovencitas de 17 años o menos, lo cual nos da un total de $3.4 \%$ de estas pacientes atendidas.

Distribuidas por edad obtuvimos una media de 16.25 con d.e. de 1 y rangos entre los 13 y 17 años (Tabla 1).

Tabla 1

EDAD DE LAS PACIENTES

\begin{tabular}{|llc|}
\hline Edades & No. de Adolescentes Embarazadas & $\%$ \\
\hline 13 años & 2 & 2 \\
14 años & 6 & 5.8 \\
15 años & 11 & 10.8 \\
16 años & 28 & 27.5 \\
17 años & 55 & 53.9 \\
\hline
\end{tabular}

Grafica 1

\section{LUGAR DE PROCEDENCIA}

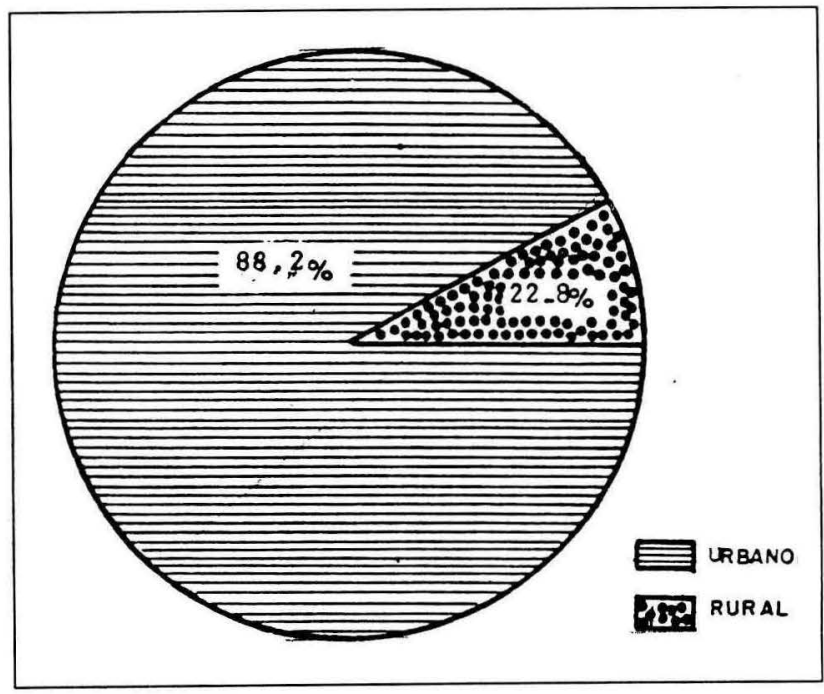

Noventa de las pacientes ( $88.2 \%$ procedían del área urbana y las restantes $12(22.8 \%$ ) del área rural (Gráfica 1$)$.

\section{Lugar de Procedencia}

El nivel socioeconómico era malo en un $21 \%$, regular en un $55 \%$ y bueno en un $21 \%$, no fue reportado en un $3 \%$, asimismo el estado civil predominante fue la unión libre con el $42 \%(n=43)$, luego las solteras con $27.4 \%(n=28)$ y casadas el $15.6 \%(n=16)$, en 15 pacientes el dato no fue reportado. La escolaridad fue bastante baja y es así como se reporta primaria en $39.6 \%$, secundaria en $37 \%$, aunque cabe anotar que casi todas con los estudios incompletos, universitaria sólo un $2 \%$ y no se encontró datos en un $21 \%$ (Tabla 2)

Tabla 2

\section{NIVEL SOCIOECONOMICO, ESTADO CIVIL Y ESCOLARIDAD}

\begin{tabular}{|l|l|l|}
\hline Nivel socioeconómico & Estado civil & Escolaridad \\
\hline Bajo 21\% & Uniónlibre 42\% & Primaria39.6\% \\
Regular 55\% & Solteras 27.4\% & Secundaria37\% \\
Bueno 21\% & Casadas 15.6\% & Universitaria 2\% \\
No fue reportado 3\% & No fue reportado 15\% & $\begin{array}{l}\text { No se encontró } \\
\text { dato 21\% }\end{array}$ \\
\hline
\end{tabular}

La edad promedio de la menarquia fue de 12.2 años con una d.e. de 1.3 y rangos entre los 10 y los 15 años (Tabla 3 ).

Tabla 3

\section{DISTRIBUCION DE LA PRIMERA MENSTRUACION EN EMBARAZADAS ADOLESCENTES SEGUN EDAD}

\begin{tabular}{|c|c|c|}
\hline Menarquia & No. de Adolescentes Embarazadas & $\%$ \\
\hline 10 años & 11 & 10.6 \\
\hline 11 años & 18 & 17.6 \\
\hline 12 años & 33 & 32.4 \\
\hline 13 años & 26 & 25.5 \\
\hline 14 años & 8 & 7.8 \\
\hline 15 años & 6 & 5.8 \\
\hline Total & 102 & $100 \%$ \\
\hline
\end{tabular}


El inicio de vida sexual (Tabla 4), fue a los 15.2 años, con rangos entre 13 y 17 años y una d.e. de 1.11 de las pacientes $(10.7 \%)$ eran secudigestantes y el resto $(89.3 \%)$ primíparas.

Tabla 4

INICIO DE VIDA SEXUAĹL POR EDADES

\begin{tabular}{|l|c|l|}
\hline Edad & $\begin{array}{c}\text { No. de Adolescentes } \\
\text { Embarazadas }\end{array}$ & $\%$ \\
\hline 13 años & 9 & 8.8 \\
14 años & 15 & 14.7 \\
15 años & 16 & 15.7 \\
16 años & 45 & 44.1 \\
17 años & 3 & 2.9 \\
No reportada & 14 & 13.7 \\
\hline Total & 102 & 100 \\
\hline
\end{tabular}

$\mathrm{X} 15.2$ años.

La edad gestacional al momento del parto fue de 13 pacientes entre las 28 y 36 semanas, y 81 entre las 37 y 42 semanas, no fue reportada la edad gestacional en ocho de las pacientes (Tabla 5), pero los bebés pesaron más de 3 $\mathrm{kg}$., teniendo un peso promedio de los mismos en 3.239 gr., con d.e. de 643 gr. y rangos entre los 1.260 y los 4.200 grs.; 9 de los recién nacidos pesaron menos de 2499 grs. $(8.8 \%)$.

Tabla 5

DISTRIBUCION SEGUN EDAD GESTACIONAL

\begin{tabular}{|l|c|c|}
\hline Edad Gestacional & $\begin{array}{c}\text { No. de Adolescentes } \\
\text { Embarazadas }\end{array}$ & $\%$ \\
\hline Pre-Término: $28-36$ sem. & 13 & 12.7 \\
A - Término: $37-42$ sem. & 81 & 79.4 \\
No registrada & 8 & 7.8 \\
\hline Total & 102 & $100 \%$ \\
\hline
\end{tabular}

La vía del parto más frecuente (Tabla 6) fue la vía vaginal con $85.5 \%$ partos eutócicos y $3.9 \%$ intervenidos (espátulas o fórceps), para un total de $89.4 \%$ de partos por vía vaginal y $10.6 \%$ de los casos $(n=11)$ fueron resueltos por cesárea; siendo la primera causa la desproporción céfalo-pélvica, $(n=4)$, la segunda el sufrimiento fetal agudo $(n=2)$; también se presentó como indicación un caso de ruptura de membranas sin respuesta a la inducción, un bebé en pélvica y otro en transversa, una de las pacientes con desproporción además tenía condilomatosis vaginal severa.

$\mathrm{Al}$ analizar la actitud asumida por las jóvenes madres al saberse embarazadas el $30.4 \%(n=31)$ manifestó alegría, el $32.3 \%$ manifestó indiferencia $(n=33)$ el $26.5 \%$ $(\mathrm{n}=27)$ manifestó angustia y desespero y el resto no fue reportado.
Tabla 6

VIA DEL PARTO

\begin{tabular}{|l|c|r|}
\hline Vía del parto & $\begin{array}{c}\text { No. de Adolescentes } \\
\text { Embarazadas }\end{array}$ & $\%$ \\
\hline Vaginal & 87 & 85.5 \\
Cesárea & 11 & 10.6 \\
Vaginal & 4 & 3.9 \\
intervenido & 102 & $100 \%$ \\
\hline Total & & \\
\hline
\end{tabular}

Entre las complicaciones más frecuentes (Tabla 7) tuvimos el parto prematuro con 13 pacientes (12.7\%), la ruptura prematura de membranas con $4 \%$ lo cual equivale a 4 pacientes, toxemia del embarazo en $2 \%$ de los casos, desgarros transpartos en 4 pacientes $(4 \%)$ y retención de placenta en 2 . Cabe anotar que un $75 \%$ de las pacientes no tuvieron complicaciones.

Tabla 7

\section{COMPLICACIONES PRENATALES Y PUERPERALES EN EMBARAZADAS ADOLESCENTES}

\begin{tabular}{|l|c|r|}
\hline $\begin{array}{c}\text { Complicaciones } \\
\text { Puerperales y Prenatales }\end{array}$ & $\begin{array}{c}\text { No. de Adolescentes } \\
\text { Embarazadas }\end{array}$ & $\%$ \\
\hline Parto prematuro & 13 & 12.7 \\
Desgarros & 4 & 4 \\
Ruptura prematura & & 4 \\
de membranas ovulares & 4 & 4.8 \\
Sufrimiento fetal agudo & 3 & 2 \\
Retención de placenta & 2 & 2 \\
Enfermedad hipertensiva & 2 & 75.5 \\
del embarazo & 77 & 100 \\
\hline Ninguna & 102 & \\
\hline Total & & \\
\hline
\end{tabular}

El sexo de los productos se distribuyó en $54.8 \%$ masculino y $45.2 \%$ femenino. No tuvimos un solo caso de mortalidad materna o perinatal, y todos los prematuros evolucionaron satisfactoriamente.

\section{Discusión}

Aunque obtuvimos un total de $5.8 \%$ de pacientes menores o con 17 años atendidas en el Hospital Universitario Metropolitano por aborto $(2.3 \%)$ o por parto $(3.5 \%)$, anotamos que la incidencia real de embarazo y aborto en adolescentes es mayor, pues al incluir aquellas de 18,19 y 20 años nos da un total de 401 casos lo cual equivale al $13.8 \%$, cifra semejante a lo publicado anteriormente (4-5).

Este grupo de pacientes no fue incluido en nuestro grupo de estudio, pues se ha demostrado que no tienen alto riesgo obstétrico, y que su comportamiento obstétrico es semejante a la mujer adulta $(6,7)$. 
Es así entonces como encontramos también que por cada embarazada con 17 años o menos, tenemos 28 mayores de esta edad (1:28). El hecho que el $88.2 \%$ de las pacientes provengan del área urbana es comprensible debido a la ubicación del Hospital Universitario Metropolitano en área central de la ciudad de Barranquilla, existiendo otros hospitales más periféricos donde probablemente acuden más pacientes del área rural; debido probablemente a esto, también obtuvimos un total de $77.4 \%$ con estatus socioeconómico entre regular y bueno a diferencia de otros estudios $(5,7)$ donde esta incidencia es de 65 y $60 \%$ de área marginada respectivamente.

La escolaridad concuerda con publicaciones anteriores $(2,8,9)$ en la cual la mayoría de estas jóvenes se encuentran o estudiando o con estudios incompletos por el mismo embarazo. Está demostrado que entre más tempranamente es la menarca, el desarrollo pélvico aumenta (4) y también se ha encontrado que ésta es cada vez a menor edad, en nuestro grupo de pacientes fue a los 12.2 años y en trabajos anteriores reportan 12.4 años $(4-5,10)$.

El inicio de vida sexual se realizó a la edad promedio de 15.2 años, lo cual, aunque está ligeramente más alto que otros trabajos (5), corresponde al promedio para la clase social media, según otras publicaciones (9).

Como es obvio, la mayoría de las pacientes eran primigestantes, y nos produjo satisfacción a pesar de ser estas madres adolescentes, que el número total de bebés con peso menor de 2499 gr. fue de sólo 9 partos (8.8\%) y correspondían no a bajo peso, sino que estas pacientes tuvieron su parto antes de las 37 semanas, estando parecido a lo publicado anteriormente por Barrios (5), Semmens (11), López (12) y Barbero (13), los cuales dan cifras desde el $9 \%$ hasta $10.4 \%$ de prematuros, y esto forma parte de las complicaciones totales, las cuales suman un $25 \%$ siendo el parto prematuro predominante con 13 pacientes y consideramos es debido en su gran parte al mal control prenatal, a la inmadurez socio-afectiva y a la preparación inadecuada de estas jóvenes adolescentes para atender la importancia del embarazo y el parto, así como también influyen la desnutrición, baja estatura, analfabetismo, consumo de drogas, infecciones, etc., $(6,12,15)$ de donde recalcamos lo vital que resulta que este grupo de pacientes sea atendido de manera multidisciplinaria, con la participación de psicología, nutrición, médico etc., y asimismo motivarlas para que asistan a los cursos de preparación para parto y el que exista una consulta gineco-obstétrica especializada en adolescentes, para lo cual remito al excelente trabajo publicado por Duarte (16) en 1981.

\section{Conclusiones}

Tenemos en nuestro medio una incidencia de $13.8 \%$ de embarazos en menores de 20 años y de $5.8 \%$ en adolescentes de 17 años y menos.

El $82.2 \%$ de las pacientes proceden del área urbana y el $77.4 \%$ son de nivel socioeconómico medio.

El inicio de vida sexual en este grupo de pacientes fue a los 15.2 años en promedio y la menarquia a los 12.2 años en promedio.

Hubo un total de $25 \%$ de complicaciones siendo la más frecuente el parto prematuro.

Es importante que existan en todas las instituciones hospitalarias, una consulta especializada para atender a las adolescentes embarazadas, como también para realizar ginecología infanto-juvenil, integrada por un equipo multidisciplinario de salud (médico, psicólogo, enfermera, nutricionista, trabajadora social, fisioterapeuta, etc.).

\section{BIBLIOGRAFIA}

1. Martínez P. Concepto de adolescencia. Selecta Médica 1991; 2(4): 25-27

2. Rico De Alonso A. Madres solteras adolescentes. Editorial Plaza y Janes. Santafé de Bogotá, Colombia. 1986.

3. Franco G. El adolescente colombiano. Memorias II curso de atención integral al adolescente. Subdirección de salud CAFAM. Santafé de Bogotá. 1989; 29-35.

4. Duarte-Contreras A. Ginecología de la niña y de la adolescente. Editorial Salvat Colombiana. Santafé de Bogotá, Colombia. 1988; 443-461.

5. Barrios Amaya J. Aspectos perinatales de las adolescentes. Rev. Colombiana de obstetricia y ginecología. 1985; 36: 102-112.

6. Sweeney P. A comparison of low birth weight, perinatal mortality, and infant mortality between first and second birth to women 17 years and younger. Am. J. Obstet. Gynecol. 1989; 160: 1361-1370.

7. Coll A., Baldi E., Casavilla F. Diagnóstico de la situación perinatal en la adolescente embarazada. Obstet. Ginecol. Lat. Am. 1986; 44: 205-210.

8. Duarte de Useche C. Estudio descriptivo de adolescentes embarazadas. Subdirección de salud. CAFAM. Santafé de Bogotá. 1989; 57-61.
9. Duarte-Contreras A., Barreto-López A. Factores determinantes del embarazo en adolescentes solteras. Rev. Col. Obstet. Ginecol. 1985; 36: 291-305.

10. Acosta Osio G., Franco N. Síndrome de tensión premenstrual, estudio de 302 casos. Rev. Col. Obstet. Ginecol. 1992; 43: 43-49.

11. Semmens J., Krantz K. El mundo del adolescente. Editorial Continental. México DF. 1973.

12. López G., Riaño G., Karen S. La embarazada adolescente. Monografías de la C.C.R.P. 1979; 14: 109-130.

13. Barbero C., de Miguel JR. Edad materna inferior a 20 años. Riesgos gravídicos y perinatales. Clin. Invest. Gin. Obstet. 1984; 11: 120-124.

14. Jhonson JW., Dubin N. Prevención del parto prematuro. Clin. Obstet. Ginecol. Editorial Interamericana. México DF. 1980; 1: 55-56.

15. McAnarney E. Embarazo y maternidad en adolescentes: nuevos datos, nuevos problemas. Pediatrics. 1985; 19: 283-284.

16. Duarte-Contreras A. Bases para la atención ginecológica especializada a niñas y adolescentes. Rev. Col. Obstet. Ginecol. 1981; 32: 299-311. 